\title{
ALIMENTAÇÃO DE CRIANÇAS EM AMBIENTE ESCOLAR - UMA VISÃO INTERDISCIPLINAR
}

\section{CHILDREN'S NUTRITION IN SCHOOL ENVIRONMENT - AN INTERDISCIPLINARY VIEW}

\section{ALIMENTACIÓN DE NIÑOS EN AMBIENTE ESCOLAR - UNA VISIÓN INTERDISCIPLINAR}

Klismam Marques dos Santos ${ }^{* 1}$, Maria Laura Martins ${ }^{1}$, Larissa C. da S. Marinho $^{1}$, Solimar A. G. V. Sobrinho ${ }^{1}$, Icaro L. de Carvalho Alves ${ }^{1}$, Marta A. dos Santos ${ }^{2}$.

${ }^{1}$ Discente do curso de Nutrição da Universidade Federal do Tocantins, Palmas, Tocantins, Brasil.

${ }^{2}$ Docente do curso de Nutrição da Universidade Federal do Tocantins, Palmas, Tocantins, Brasil.

*Correspondência: Curso de Nutrição-Universidade Federal do Tocantins, Av. NS 15, 109 Norte, Palmas,

Tocantins, Brasil. CEP:77.010-090.e-mail klisman1995@hotmail.com.

\section{Artigo recebido em 12/08/2018 aprovado em 12/06/2019 publicado em 13/06/2019.}

\section{RESUMO}

Este estudo tem como objetivo a realização de um levantamento das características da alimentação escolar e como isso influencia no desenvolvimento biopsicossocial das crianças. Trata-se de uma revisão bibliográfica baseada na leitura de artigos científicos selecionados através dos bancos de dados Google Acadêmico e BVSalud, além de utilizar a Política Nacional de Alimentação e Nutrição (PNAN), Política Nacional de Promoção à Saúde e o Guia Alimentar para a População Brasileira. Os estudos encontrados relacionados ao assunto mostram a importância de uma boa influência na alimentação no período da infância, pois é nessa fase onde há transformações biopsicossociais e onde a criança adquire grande parte dos seus hábitos alimentares, o ambiente escolar é de extrema importância, visto que é onde a criança passa grande parte do seu tempo. A participação do professor e dos outros profissionais que estão presentes no ambiente escolar podem auxiliar a criança a construírem bons hábitos nutricionais.

Palavras-chave: Alimentação escolar, crianças, interdisciplinaridade.

\section{ABSTRACT}

This study aims to perform a survey of the characteristics of school feeding and how this influences the biopsychosocial development of children. This is a bibliographic review based on the reading of scientific articles selected through the Google Academic and BVSalud databases, in addition to using the National Policy on Food and Nutrition (PNAN), National Health Promotion Policy and the Food Guide for Brazilian population. The studies found related to the subject show the importance of a good influence in the feeding in the period of the childhood, since it is in that phase where there are biopsicossocial transformations and where the child acquires great part of its feeding habits, the school environment is of extreme importance, since is where the child spends much of his time. The participation of the teacher and other professionals who are present in the school environment can help the child with his good nutrition habits.

Keywords: School feeding, children, interdisciplinarity.

\section{RESUMEN}

Este estudio tiene como objetivo la realización de un levantamiento de las características de la alimentación escolar y cómo ello influye en el desarrollo biopsicosocial de los niños. Se trata de una revisión bibliográfica basada en la lectura de artículos científicos seleccionados a través de los bancos de datos Google Académico y BVSalud, además de utilizar la Política Nacional de Alimentación y Nutrición (PNAN), Política Nacional de Promoción a la Salud y la Guía Alimentaria para la Población Brasileña. Los estudios encontrados relacionados al tema muestran la importancia de una buena influencia en la alimentación en el período de la infancia, pues es en esa fase donde hay transformaciones biopsicosociales y donde el niño adquiere gran parte de sus hábitos alimenticios, el ambiente escolar es de extrema importancia, ya que es donde el niño pasa gran parte de su tiempo. La participación del 
profesor y de los otros profesionales que están presentes en el ambiente escolar pueden ayudar al niño a construir buenos hábitos nutricionales.

Descriptores: Alimentación escolar, niños, interdisciplinariedad.

\section{INTRODUÇÃO}

A infância é uma fase marcada por transformações biopsicossociais da criança, na qual são adquiridos os hábitos alimentares, por meio de alterações de apetite e de ingestão alimentar. Assim, uma alimentação saudável e adequada é um fator determinante para o crescimento e desenvolvimento da saúde da criança, impactando nos hábitos e práticas alimentares que irão perdurar durante a vida adulta, além de prevenir doenças associadas à má alimentação, diabetes tipo II, hipertensão arterial, dislipidemias e, principalmente, a obesidade, cuja prevalência entre os indivíduos em idade escolar é considerada alta (APN, 2013).

O estado nutricional com predominância de obesidade entre crianças está relacionado a fatores ambientais, psicológicos e biológicos, que incluem a falta de atividade física, consumo de alimentos de alta densidade calórica, perfil socioeconômico, estrutura genética, acesso à alimentos e à informação nutricional, entre outros, podendo ocasionar distúrbios metabólicos e mudanças na composição corporal e, inclusive, distúrbios psicossociais, como baixa autoestima, depressão, isolamento social, etc (ARAÚJO et al., 2016; ALBUQUERQUE et al., 2014).

A família, como primeiro núcleo de integração social da criança, influencia diretamente no seu padrão alimentar, entretanto a partir do contato com outros grupos sociais, como escolas e creches, a criança adquire novos hábitos alimentares, alterando seu padrão e estado nutricional. Assim, no ambiente escolar, as crianças estão expostas a experiências e exemplos do seu círculo de convivência devido à intensa integração social (ACCIOLY e ELIZABETH, 2009).

Dessa forma a escola deve assumir o papel de promotora da saúde, se baseando nas políticas e programas de alimentação no planejamento e execução do cardápio da merenda escolar e dos demais alimentos oferecidos dentro da unidade escolar, visando oferecer aos seus estudantes uma alimentação de qualidade que irá suprir parcialmente as necessidades nutricionais do indivíduo, além de estimular hábitos alimentares saudáveis, por meio de boas práticas de higiene e alimentação e ações de educação alimentar (ACCIOLY e ELIZABETH, 2009).

Deve-se atentar para a composição nutricional do cardápio adotado no ambiente escolar, levando em consideração as necessidades nutricionais das crianças e seus hábitos alimentares, priorizando a oferta de refeições que incluam todos os grupos de macronutrientes e micronutrientes necessários para o adequado desenvolvimento intelectual, cognitivo e biológico do indivíduo, evitando assim alimentos de baixo valor nutricional e potencialmente prejudiciais à saúde, como açúcares, conservantes e gorduras saturadas, por exemplo (APN, 2013).

As políticas e programas voltados à alimentação e nutrição possuem papel fundamental na orientação e determinação das ações de promoção da saúde alimentar no ambiente escolar. Para tanto, as escolas devem atender às diretrizes estabelecidas pelo Ministério da Saúde na Política Nacional de Alimentação e Nutrição - PNAN, a qual propõe "respeitar, proteger, promover e prover os direitos humanos à saúde e à alimentação" (Brasil, 2012), 
articulando com a Política Nacional de Promoção da Saúde - PNPS, que estabelece um "conjunto de estratégias e formas de produzir saúde, no âmbito individual e coletivo" (Brasil, 2014) e com o Programa Nacional de Alimentação Escolar - PNAE, que oferece alimentação escolar e ações de educação alimentar e nutricional a estudantes de todas as etapas da educação básica pública (FNDE, 2006), além de contar com o auxílio do Guia Alimentar para a População Brasileira, o qual orienta a adoção de um estilo de vida saudável e de escolhas alimentares adequadas (Brasil, 2014).

No processo de aplicação das políticas públicas e construção dos hábitos alimentares no ambiente escolar, é essencial que haja a adoção da interdisciplinaridade, com o envolvimento de diversos profissionais que atuarão de forma a interferir positivamente sobre as práticas alimentares e no apoio psicossocial às crianças dentro da unidade escolar, contribuindo para a melhoria do estado nutricional infantil, promoção da educação nutricional e redução dos agravos à saúde relacionados à má alimentação, impactando não apenas na fase infantil, mas durante toda a vida do indivíduo, tanto no ambiente interno da escola, quanto nos ambientes externos, como o ambiente familiar. Esses profissionais incluem não apenas nutricionistas, como também educadores, psicólogos, enfermeiros, merendeiros, educadores físicos, entre outros (ACCIOLY e ELIZABETH, 2009).

O objetivo desta revisão é realizar um levantamento referente às características da alimentação escolar à qual as crianças são submetidas e de que forma tais práticas e hábitos alimentares no ambiente escolar influenciam no seu desenvolvimento biopsicossocial.

\section{MATERIAIS E MÉTODOS}

Este trabalho constitui-se de uma revisão realizada entre agosto e novembro de 2017. Para a realização do mesmo, foram consultados artigos científicos que foram designados através da procura nos bancos de dados Google Acadêmico e BVSalud além de referências como a Política Nacional de Alimentação e Nutrição - PNAN, Política Nacional de Promoção à Saúde - PNPS, Programa Nacional de Alimentação Escolar - PNAE e o Guia Alimentar para a População Brasileira.

Para a seleção dos artigos, foi realizada uma busca nos bancos de dados utilizando os descritores “alimentação escolar”, “crianças”, “alimentação saudável" e "interdisciplinaridade". Após isto, foram selecionados cinco artigos que abordavam como é a alimentação das crianças em ambiente escolar, envolvendo não somente os nutricionistas, mas também outros profissionais como psicólogos e professores.

As políticas utilizadas neste trabalho, juntamente com o guia alimentar, possuem o objetivo de diminuir as patologias relacionadas à alimentação e nutrição e promover melhorias nos modos de viver da população, contribuindo assim para a promoção da saúde.

\section{RESULTADOS E DISCUSSÃO}

A partir da utilização dos descritores acima citados, foi encontrado um total de 20 estudos. Após descarte dos resumos duplicados, a leitura dos resumos e a aplicação dos critérios indicados resultaram uma amostra final de 5 estudos, selecionados entre 2006 e 2017. Foram consultados estudos publicados em periódicos nacionais que tivessem como objeto a alimentação de crianças nas escolas. O método qualitativo foi o mais empregado entre os artigos e dissertações presentes na amostra final, e, análise documental e entrevistas, as técnicas e instrumentos mais utilizados. 
O estudo de da Silva, Margareth Xavier, et al (2014). considerou importante a participação dos pais e o desenvolvimento de atividades educativas com os escolares. Realizou-se reunião entre os responsáveis pelos alunos e o grupo da pesquisa, utilizando como base o Guia Alimentar (SICHIERI, NASCIMENTO e MOURA, 2002), considerou-se que o apoio dos pais às possíveis escolhas que essas crianças fariam, após a participação nas atividades educativas a respeito de alimentos e de alimentação saudável, poderia trazer efeito positivo no que diz respeito à alimentação da família.

Com relação aos 10 passos para alimentação saudável e sua aplicação em escolas Ana Paula WEBER, et al (2015). Realizou um estudo intitulado “Adesão aos '10 passos da alimentação saudável' para crianças" e fatores associados em escolares, estudo de caraterística transversal com 813 escolares do $1^{\circ}$ ano do Ensino Fundamental de São Leopoldo (RS). Foram aplicados questionários às mães/responsáveis com questões sobre alimentação, atividade física, tempo de tela, e características sociodemográficas. Este estudo teve como objetivo avaliar a frequência de adesão aos “10 Passos da Alimentação Saudável para Crianças" e seus fatores associados em escolares. Onde se foi possível observar percentual baixo de adesão às recomendações para a grande maioria dos passos e um baixo número de passos atingido. Tais resultados são preocupantes visto que os hábitos desenvolvidos na infância tendem a permanecer na adolescência e consequentemente na vida adulta, e maus hábitos alimentares têm sido correlacionados com obesidade e fatores de risco cardiometabólicos.

Em seu estudo intitulado Qualidade da dieta de pré-escolares de 2 a 5 anos residentes na área urbana da cidade de Pelotas, RS, Katharine Konrad Leal, et al (2015). Procura apresentar como as preferências alimentares das crianças são adquiridas por meio de experiências repetidas do consumo de determinados alimentos. Tais hábitos são reflexos de sua ingestão alimentar, acostumada às condições fisiológicas e ao contexto social em que a criança está inserida. Levando em consideração que nesta fase as crianças têm preferência por alimentos com maior porte calóricos, pois trazem maior saciedade e consequentemente o aporte energético necessário para as suas necessidades básicas.

Este estudo, de base populacional, feito em município de porte médio da Região Sul do Brasil, apontou que a dieta das crianças estudadas necessita de melhorias, inicialmente foram aplicados questionário de frequência alimentar (QFA), o QFA foi validado com o uso de três recordatórios de 24 horas e a análise da qualidade da dieta foi feita por meio do Índice de Alimentação Saudável (IAS). Cerca de dois terços das crianças (64\%) atingiram escore máximo no grupo dos cereais ao ingerir quatro porções diárias, em relação ao consumo de legumes e verduras, apenas $45,7 \%$ das crianças atingiram a recomendação preconizada pela IAS, que é de duas porções diárias para meninos e uma para meninas, o inverso foi observado para o consumo adequado de frutas (duas porções diárias para meninos e uma porção para meninas), visto que $86 \%$ das crianças atingiram a recomendação. Foi observado um considerável consumo de leite e derivados, já que $81,1 \%$ das crianças atingiram a recomendação para esse grupo, que é de duas porções diárias para meninos e uma para meninas. Quanto ao consumo de carnes e ovos, $85,4 \%$ das crianças consumiram o recomendado (uma porção diária).

As leguminosas, grupo avaliado por meio do consumo de feijão, são importantes fontes de ferro, ácido fólico e fibras alimentares e estiveram presentes na alimentação diária de 89,2\% das crianças, as quais atingiram a pontuação máxima nesse grupo alimentar, o consumo de açúcares, balas, chocolates e salgadinhos estiveram acima do recomendado, já que 
$99,6 \%$ das crianças estudadas consumiam mais do que uma porção de alimentos desse grupo, diariamente, todas as crianças deste estudo consumiram porções do grupo dos óleos e gorduras acima da recomendação, que é de uma porção diária. Essa situação pode contribuir para o desenvolvimento de doenças crônicas não transmissíveis (DCNT). Para os componentes sódio e colesterol, $36,9 \%$ e $45,1 \%$ das crianças, respectivamente, ingeriram $\leq 2.400 \mathrm{mg}$ diárias de sódio e $\leq 300 \mathrm{mg}$ diárias de colesterol e receberam pontuação máxima, o consumo frequente e em grande quantidade de gorduras e sal aumenta o risco de doenças como obesidade, hipertensão arterial e doenças do coração e em relação à variedade da dieta, os resultados apontaram para uma dieta variada, já que $97,3 \%$ das crianças receberam mais de sete pontos nesse componente por terem consumido sete ou mais tipos de alimentos diariamente.

No estudo intitulado "Perfil socioeconômico e nutricional de escolares em uma instituição pública de ensino em Jaguaribara-CE", ROSA, Samara Almeida Mesquita, etal (2017), procura apresentar o perfil socioeconômico e como o mesmo está intimamente ligado com o perfil nutricional dos escolares, com o objetivo avaliar o estado nutricional e características socioeconômicas de crianças escolares matriculadas em uma instituição pública de ensino em Jaguaribara, Ceará. O estudo apresenta característica transversal, descritivo, analítico e quantitativo, que foi desenvolvido com a finalidade de estabelecer o diagnóstico nutricional de crianças em uma escola municipal. A coleta de dados foi realizada por meio da aplicação de um questionário socioeconômico e avaliação nutricional.

No presente estudo foi possível observar maior percentual de crianças que não apresentam boa nutrição e uma alimentação de qualidade, com renda superior a 2 salários mínimos, apesar de $90 \%$ dos avaliados terem renda inferior a esse valor, o que reforça uma mudança no perfil nutricional da população, em que o sobrepeso/obesidade e um malefício que vem afetando as crianças com diversas faixas de renda, reforçando a importância de se considerar, também, os fatores comportamentais das mesmas. Na pesquisa cerca de $43,66 \%$ das crianças não praticam nenhum tipo de atividade física. Sabe-se que é primordial cultivar um estilo de vida saudável desde a infância, pois é de grande valia para a prevenção da obesidade, visto que muitas características da fase adulta são adquiridas e ou consolidadas na infância, tornando de suma importância a avaliação dos fatores relacionados com os hábitos alimentares e prática de atividade física.

Com relação à visão das crianças no que diz respeito à alimentação saudável, Olga Maria Ramalho de Albuquerque, et al (2014). Apresenta seu estudo intitulado, 'Percepção de estudantes de escolas públicas sobre o ambiente e a alimentação disponível na escola: uma abordagem emancipatória'". O estudo objetiva analisar a percepção dos escolares da rede pública sobre o ambiente escolar e a alimentação disponível na escola. Foram realizadas Oficinas em Dinâmica de Grupos (ODG) com estudantes das escolas públicas de Ceilândia-DF. Ao final de cada dinâmica, as crianças escreveram cartas aos pais e educadores com sugestões de mudança para o esboço de um ambiente que tenha com foco facilitar escolhas mais saudáveis, as quais foram submetidas à análise de conteúdo. Nos resultados foi possível evidenciar o entendimento dos escolares no reconhecimento de uma alimentação saudável, além do discernimento para as razões que evidenciam e afirmam as escolhas não saudáveis. As crianças apresentaram completa astucia com relação à incoerência dos tomadores de decisão e educadores que fazem recomendações para um consumo saudável, mas não proporcionam meios para o acesso desses alimentos nas escolas. 
De acordo com as crianças, não existe na escola a base necessária para que se façam escolhas benéficas. $\mathrm{O}$ autor coloca que a necessidade de se avaliar as práticas dos educadores e de profissionais de saúde com o objetivo de intensificar a participação ativa das crianças na criação de propostas a serem aplicadas nas escolas. Sendo de extrema importância escutá-las para a edificação de um ambiente saudável na escola, visto que são protagonistas e ativadoras dos processos de mudança, pois, estando os mesmos inseridos no ambiente escolar, conseguem identificar as dificuldades presentes e as escolhas saudáveis.

Na contemporaneidade, a Política Nacional de Alimentação e Nutrição - PNAN, preconiza que o conjunto de ações de Promoção de Alimentação Adequada e Saudável - PAAS, aliado às demais ações de promoção da saúde acarreta possibilidades inovadoras que contribuem de forma socialmente positiva para o desenvolvimento dos indivíduos e comunidades (PNAN, 2012).

A PNAN tem por objetivo proporcionar a melhoria das condições de alimentação, nutrição e saúde da população brasileira, por meio de ações voltadas a promoção de práticas alimentares adequadas e saudáveis nas diferentes faixas etárias e ambientes sociais, vigilância alimentar e nutricional e a prevenção de agravos relacionados à alimentação e nutrição.

Em conformidade com o que foi explanado a respeito da realidade dos escolares, é de extrema importância à intensificação de ações no âmbito da educação nutricional, levando informações de forma lúdica e didática para formação dessas crianças a respeito de suas próprias escolhas alimentares, são recomendadas melhorias na alimentação servida nas escolas de modo a apresentar uma alimentação saudável, variada e que atenda às necessidades nutricionais das crianças em pleno desenvolvimento, de acordo com os 10 passos para alimentação saudável. Importante também a abordagem em um dos artigos, sobre o consumo de alimentos in natura, alertando que este deve ser sempre priorizado para que se tenha uma boa nutrição. Além da redução no consumo de gorduras e doces, visto o alto índice de crianças que os consomem acima da recomendação.

Sob o mesmo ponto de vista, a Política Nacional de Promoção da Saúde - PNPS, que tem como objetivo promover a equidade e a melhoria das condições e dos modos de viver, de forma a ampliar a potencialidade da saúde individual e coletiva e reduzindo riscos e vulnerabilidades a saúde que decorrem dos determinantes sociais, econômicos, políticos, culturais e ambientais, tem de ser atrelada às intervenções que devem ser realizadas no âmbito escolar e social (PNPS, 2014).

Deste modo, podendo ser elaboradas e aplicadas políticas públicas que visem à conscientização da população com relação à importância da alimentação na promoção e prevenção de agravos na saúde, que se estendem desde depressão a doenças crônicas degenerativas como a diabetes, com ênfase no objetivo de transmudar o alto índice de obesidade no país, que é, atualmente, um dos maiores problemas de saúde pública. Tais medidas são essenciais para que se tenha uma vicissitude nos hábitos alimentares da população brasileira, melhorando os mesmos desde cedo e, consequentemente, perpetuar esses novos costumes para as próximas gerações.

\section{CONCLUSÃO}

Através dos estudos analisados foi possível observar que o ambiente escolar tem grande influência na construção da personalidade das crianças, pois é um espaço onde há a obtenção de valores e conhecimentos que podem auxiliar na mudança de hábitos, evitando casos futuros de obesidade e doenças relacionadas a alimentação. A merenda escolar pode contribuir 
significativamente para a melhoria das condições nutricionais dos alunos, diminuindo o teor de deficiências nutricionais. A escola pode ser usada como um local de promoção da saúde, onde os discentes podem transmitir conhecimento por meio de ações de educação em saúde.

Na escola, é possível utilizar da influência dos professores para a realização das ações de educação em saúde, como exemplo pode-se citar o professor de educação física, que pode utilizar das suas aulas práticas para influenciar o aluno a praticar exercícios, além disso, os outros professores podem organizar ações que envolvam a importância de uma alimentação saudável.

Todos os autores declararam não haver qualquer potencial conflito de interesses referente a este artigo.

\section{REFERÊNCIAS}

APN - ASSOCIAÇÃO PORTUGUESA DE NUTRICIONISTAS. Direção-Geral do Consumidor. Governo de Portugal (Ed.). Alimentação em Idade Escolar: Guia Prático para Educadores. 2013. Disponível em: <http://www.apn.org.pt>. Acesso em: 21 nov. 2017.

ARAÚJO, C.G; ROSA, S.A.M. Perfil socioeconômico e nutricional de escolares em uma instituição pública de ensino em Jaguaribara - CE. Rev. Aps, Juiz de Fora: UFJF, v. 4, n. 19, p.602-612, out./dez. 2016.

ACCIOLY, E. A escola como promotora da alimentação saudável. Rev. Ciência em Tela, Rio de Janeiro: UFRJ, v. 2, n. 2, p.1-9, jul./dez. 2009.

ALBUQUERQUE, O.M.R; MARTINS, A.M; MODENA, C.M; CAMPOS, H.M. Percepção de estudantes de escolas públicas sobre o ambiente e a alimentação disponível na escola: uma abordagem emancipatória. Saúde e Sociedade, [s.1.], v. 23, n. 2, p.604-615, jun. 2014.
BRASIL. Ministério da Saúde. Guia alimentar para a população brasileira. 2. ed., 1. reimpr. Brasília : Ministério da Saúde, 2014.

BRASIL. Ministério da Saúde. Política Nacional de Alimentação e Nutrição. Brasília: Ministério da Saúde, 2012.

BRASIL. Ministério da Saúde. Política Nacional de Promoção da Saúde. Brasília: Ministério da Saúde, 2014.

Brasil. Resolução/CD/FNDE n ${ }^{\circ} 38$ de 16 de julho de 2009. Dispõe sobre o atendimento da alimentação escolar aos alunos da educação básica no Programa Nacional de Alimentação Escolar (PNAE). Diário Oficial da União 2009; 17 jun.

DA SILVA, M.X; SERAPIO, J; PIERUCCI, A.P.T.R; PEDROSA, C. Nutrição escolar consciente: estudo de caso sobre o uso de oficinas de culinária no ensino fundamental. Ciências \& Cognição, v. 19, n. 2, 2014.

FUNDO NACIONAL DE DESENVOLVIMENTO DA EDUCAÇÃO (FNDE). Resolução no 32 de 10 de agosto de 2006. Estabelece normas para a execução do Programa Nacional de Alimentação Escolar - PNA. Brasília. 2006.

LEAL, K. K.; SCHNEIDER, B. C.; FRANÇA, G. V. A.; GIGANTE, D. P.; SANTOS, I.; ASSUNÇÃO, M. C. F. Qualidade da dieta de pré-escolares de 2 a 5 anos residentes na área urbana da cidade de Pelotas, RS. Revista Paulista de Pediatria, v. 33, n. 3, p. 310317, 2015.

ROSA, S. A. M.; ARAÚJO, C. G.. PERFIL SOCIOECONÔMICO E NUTRICIONAL DE ESCOLARES EM UMA INSTITUIÇÃO PÚBLICA DE ENSINO EM JAGUARIBARA-CE. Revista de APS, v. 19, n. 4, 2017.

WEBER, A.P; HENN, R.L; VICENZI, K; PANIZ, V.M.V; OLINTO, M.T.A. Adesão aos" 10 passos da alimentação saudável para crianças" e fatores associados em escolares.. Revista de Nutrição, v. 28, n. 3, p. 289-304, 2015. 